EPJ Web of Conferences 59, 04006 (2013)

DOI: $10.1051 /$ epjconf/20135904006

(C) Owned by the authors, published by EDP Sciences, 2013

\title{
Numerical analysis of anisotropic diffusion effect on ICF hydrodynamic instabilities
}

\author{
M. Olazabal-Loumé ${ }^{1, a}$ and L. Masse ${ }^{2}$ \\ 1 CELIA, UMR5107 Université Bordeaux I-CNRS - CEA, Université Bordeaux I, \\ 33405 Talence, France \\ 2 CEA, DAM, DIF, 91297 Arpajon, France
}

\begin{abstract}
The effect of anisotropic diffusion on hydrodynamic instabilities in the context of Inertial Confinement Fusion (ICF) flows is numerically assessed. This anisotropy occurs in indirect-drive when laminated ablators are used to modify the lateral transport $[1,2]$. In direct-drive, non-local transport mechanisms and magnetic fields may modify the lateral conduction [3]. In this work, numerical simulations obtained with the code PERLE [4], dedicated to linear stability analysis, are compared with previous theoretical results [1]. In these approaches, the diffusion anisotropy can be controlled by a characteristic coefficient which enables a comprehensive study. This work provides new results on the ablative RayleighTaylor (RT), ablative Richtmyer-Meshkov (RM) and Darrieus-Landau (DL) instabilities.
\end{abstract}

\section{INTRODUCTION}

A large number of papers published over the last 30 years have been devoted to the instability developing at the ablation front. Understanding the mechanism at work and controlling the perturbation growth are indeed, in the ICF context, key issues. It has been shown that the main stabilizing effect of the ablation front instability can be understood as a transversal diffusive mechanism [1,7]. This understanding sheds new light on the ablation front instability and in particular explains the effect of anisotropic diffusion on the perturbation growth, but also illuminates ablative RM and DL instabilities.

Main theoretical results [1] are reminded in Sect. 2. Sections 3, 4 and 5 are respectively dedicated to simulations of ablative RT, ablative RM and DL instabilities.

\section{ANISOTROPIC THERMAL DIFFUSION}

\subsection{Self-consistent theory}

According to [5-9] the linear growth rate of the ablative RT instability for large Froude numbers and large density ratio $(a \gg 1)$ is given by the relation

$$
\sigma=\sqrt{k g-a k^{2} V_{a}^{2}+4 k V_{a}}-2 k V_{a}
$$

where $g$ is the acceleration, $V_{a}$ the ablation velocity, $a$ the ratio between ablation front density and blow-off density, $k=2 \pi / \lambda$ the wave number and $\lambda$ the wavelength of the perturbation. To exhibit the

\footnotetext{
${ }^{a}$ e-mail: olazabal@celia.u-bordeaux1.fr
}

This is an Open Access article distributed under the terms of the Creative Commons Attribution License 2.0, which permits unrestricted use, distribution, and reproduction in any medium, provided the original work is properly cited. 

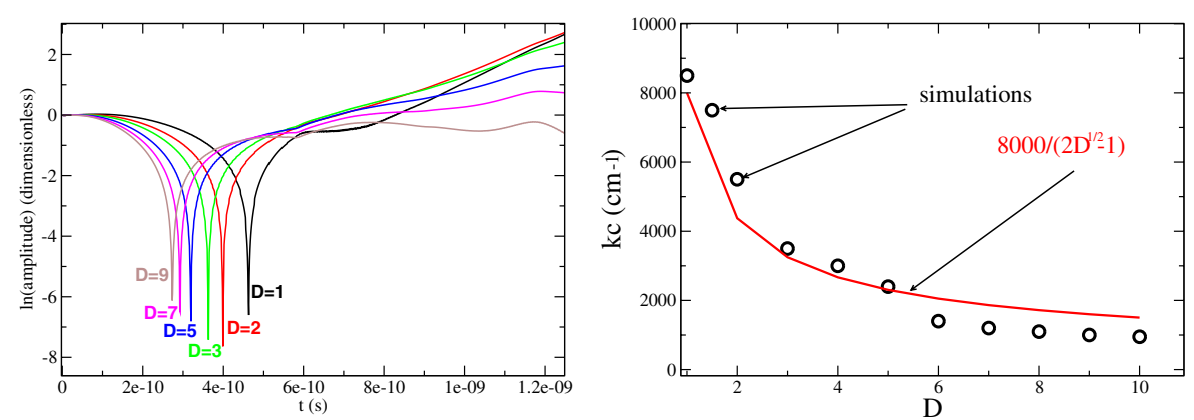

Figure 1. Left panel: stabilization of the ablative Rayleigh-Taylor instability for $\mathrm{k}=0.1 \mu \mathrm{m}^{-1}$. Right panel: cut-off wave number $k_{c}$ versus D.

stabilizing mechanism of transverse diffusion, one considers the following energy equation

$$
\rho C_{p}\left(\frac{\partial T}{\partial t}\right)=\kappa_{x}\left[\frac{\partial}{\partial x}\left(\frac{\partial T}{\partial x}\right)+D \frac{\partial}{\partial y}\left(\frac{\partial T}{\partial y}\right)\right]
$$

where $\kappa_{x}$ is the longitudinal thermal conductivity. $D=\kappa_{y} / \kappa_{x}$ is introduced to track the transverse diffusion and can be interpreted as a thermal diffusion anisotropy coefficient. With equation Eq. (2) and according to [1], equation Eq. (1) is replaced by

$$
\sigma=\sqrt{k g-a(2 \sqrt{D}-1) k^{2} V_{a}^{2}+(\sqrt{D}+1)^{2} k^{2} V_{a}^{2}}-(\sqrt{D}+1) k V_{a},
$$

where the density ratio $a$ is defined as the ratio of the ablation density to the density calculated at a distance $1 /(2 k)$ from the ablation front. The coefficient D highlights the stabilizing role of transverse thermal diffusion. The stabilization of the ablation front is consequently all the larger as the coefficient $\mathrm{D}$ is higher. We note that the cut-off wave number is proportional to $1 / \sqrt{D}$. In the ablative RM instability regime, when $g \sim 0$, the perturbation follows a damped oscillation behavior [10]. With an anisotropic diffusion, such as $D>1 / 4$, the frequency of the oscillations is

$$
\omega=k V_{a} \sqrt{a(2 \sqrt{D}-1)} .
$$

We note that, when $D$ varies, the $1 \mathrm{D}$ flow, in the $\mathrm{x}$-direction, is kept constant and is always identical to the isotropic case $D=1$.

\subsection{Numerical simulations}

We tested the theoretical predictions accuracy comparing with numerical simulations from the code PERLE. This code solves a 1D Lagrangian system including thermal conduction, laser energy deposition and a perfect gas equation of state. The bidimensional linear perturbation is developed in Fourier series. The code solves for each wavelength the corresponding linearized equations by means of specific numerical schemes. It is used to study the linear perturbation behavior of unsteady flows to understand the main physical mechanisms involved in specific situations $[4,12]$. The anisotropy diffusion coefficient $\mathrm{D}$ was introduced in PERLE for this study.

\section{ABLATIVE RAYLEIGH-TAYLOR INSTABILITY}

Simulation results are qualitatively and quantitatively consistent with theory (Eq. (3)). Numerical results presented in Fig. 1, on the left panel, concern a $60-\mu m$-thick DT foil and a maximum laser intensity $I=5 \times 10^{14} \mathrm{~W} / \mathrm{cm}^{2}$. After a shock transit time of $\sim 0.6 \mathrm{~ns}$ the foil starts to accelerate with the 


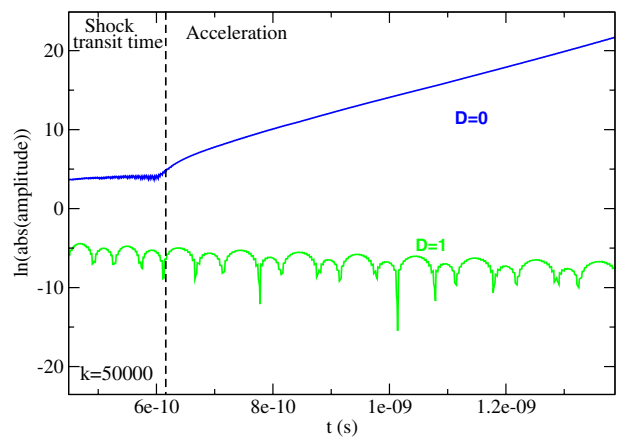

Figure 2. Suppression of stabilizing effects on the ablative Rayleigh-Taylor growth of a $5 \mu \mathrm{m}^{-1}$ wave number for $\mathrm{D}=0$.

acceleration $934 \mu \mathrm{m} / \mathrm{ns}^{2}$ and the ablation velocity $1.3 \mu \mathrm{m} / \mathrm{ns}$. A density ratio of 7 is assessed for a wave number $0.1 \mu \mathrm{m}^{-1}$. Theoretical results are calculated with Eq. (3). For $\mathrm{D}=1,2$ and 3, the growth rates given by simulations are $6.4,5.2$ and $4.4 \mathrm{~ns}^{-1}$, while theory gives $6.5,5.5$ and $4.7 \mathrm{~ns}^{-1}$. For recall, in a previous experiment [2] a coefficient $D \sim 5$ was infered. The ablation front is defined as the locus of the maximum of the space derivative of density. Growth rates are obtained using the logarithmic derivative of the amplitude of perturbation between 0.8 and 1.2 ns. Right panel of Fig. 1 shows the dependency of the cut-off wave number on the anisotropy coefficient $\mathrm{D}$.

Furthermore, simulations confirm that the main stabilizing mechanism is the transverse heat diffusion. Figure 2 presents the perturbation evolution at the ablation front for a large wave number $\mathrm{k}=5 \mu \mathrm{m}^{-1}$, for $\mathrm{D}=1$ and $\mathrm{D}=0$. In the classical case $(\mathrm{D}=1)$ this wave number is larger than the cutoff wave number and in consequence is stable. For $\mathrm{D}=0$ the wave number is unstable. No stabilization of the ablative RT instability (even for very large wave numbers) is obtained in simulations with $\mathrm{D}=0$.

\section{ABLATIVE RICHTMYER-MESHKOV INSTABILITY}

During the shock transit time (i.e. when $g=0$ ) the ablation front perturbation follows the ablative RM instability dynamics. For an anisotropic diffusion, and in the limit of short wavelengths compared to the conduction zone thickness $d_{c}$ (distance between ablation front and the critical density location ), theory predicts perturbation oscillations with a frequency given by Eq. (4) and verify $r_{D}=\frac{\omega_{D}}{\omega_{1}}=\sqrt{2 \sqrt{D}-1}$, where $\omega_{1}$ is the classical pulsation obtained for $\mathrm{D}=1$. This relation gives for examples $r_{\mathrm{D}}=2.3$ for $\mathrm{D}=10$. Left panel of Fig. 3 shows the perturbation oscillations of wave numbers $k=0.2,0.6,1 \mu \mathrm{m}^{-1}$ for $\mathrm{D}=1$ and $\mathrm{D}=10$. During the first oscillations, simulations agree with theory. This was checked for tens of simulations varying the D coefficient and the laser intensity.

After the first oscillations, the classical ablative RM theory [10] applies, independently of D. During its propagation, the shock wave generates a vorticity field in the shocked material [11]. After a given time, the perturbation evolution is then dominated by this vorticity field rather than by the ablation front dynamics itself.

\section{DARRIEUS-LANDAU INSTABILITY}

The DL instability occurs in the large wavelength limit $k d_{c} \ll 1$. When the ablation front is accelerated the DL instability is dominated by the RT instability, but during the shock transit time, when acceleration is low, the DL instability can be observed [7]. Fig. 3, right panel, presents the dispersion curves obtained 

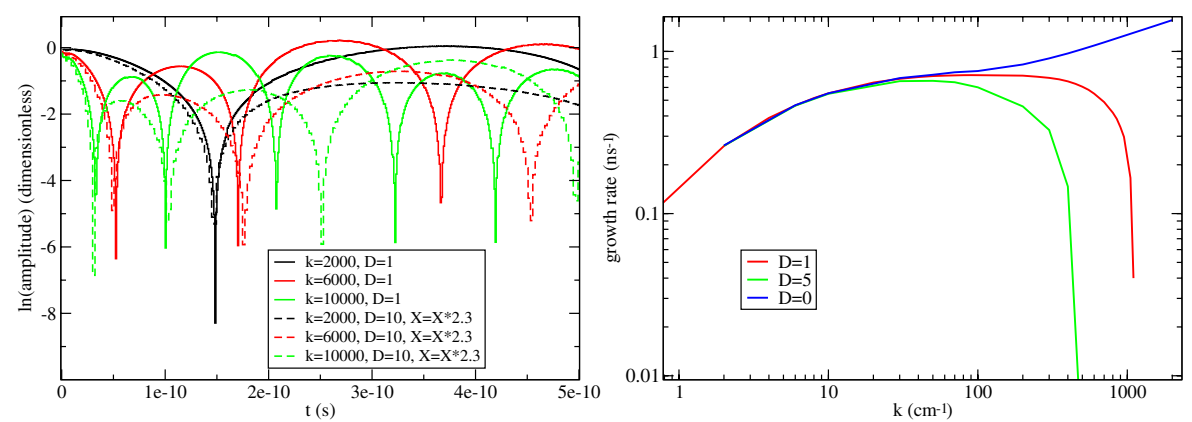

Figure 3. Left panel: perturbation oscillation frequency for $D=1,10$ and 20 . The $D=10$ curves time is renormalized using the $\mathrm{D}=1$ frequency ( 2.3 factor). In simulations, first oscillations match with anisotropic diffusion theory. Right panel: dispersion curves of the Darrieus-Landau instability for $\mathrm{D}=0,1,5$.

from simulations for a $400 \mu \mathrm{m}$-thick DT foil, a laser intensity $I=10^{13} \mathrm{~W} / \mathrm{cm}^{2}$ and D $=0,1,10$. All the growth rates were measured during the shock transit time. For $\mathrm{D}=1$, the corresponding cut-off wave number is $k=0.11 \mu \mathrm{m}^{-1}$ leading to $k_{c} d_{c}$ around 0.15 . At low wave numbers $\mathrm{k}$, the same behavior is found for all $\mathrm{D}$ values. At high wave numbers $\mathrm{k}$, the diffusion anisotropy $(\mathrm{D}>1)$ enhances the stabilizing effect of transverse diffusion for the DL instability. For D $=0$, as in the RT instability case, the DL instability is unconditionally unstable.

\section{CONCLUSION}

Using a controlled anisotropic thermal diffusion modeling, this work shed new light on the stabilization of a wrinkled ablation front. In particular, simulation results show, in agreement with theoretical results, that transverse diffusion is the main stabilizing mechanism of ablative Rayleigh-Taylor and DarrieusLandau instabilities. Moreover, for the ablative Richtmyer-Meshkov instability, the validity domain for anisotropic diffusion theory and the important role of the shock wave are evidenced.

\section{References}

[1] L. Masse et al., Phys Rev E, 83, 055401 (2011)

[2] L. Masse, Phys. Rev. Lett., 98, 245001 (2007)

[3] G. Schurtz et al., Phys. Rev. Lett., 98, 095002 (2007)

[4] M. Olazabal-Loumé and L. Hallo, Phys. Plasmas, 14, 102705 (2007)

[5] J. Sanz, Phys. Rev. Lett., 73, 2700 (1994)

[6] V. N. Goncharov et al., Phys. Plasmas, 3, 1402 (1996)

[7] P. Clavin and L. Masse, Phys. Plasmas, 11, 690 (2004)

[8] R. Betti et al., Phys. Plasmas, 3, 2122 (1996)

[9] A. Piriz et al., Phys. Plasmas, 4, 1117 (1997)

[10] V. N. Goncharov, Phys. Rev. Lett., 82, 2091 (1999)

[11] V. N. Goncharov et al., Phys. Plasmas, 13, 102702 (2006)

[12] V. Drean et al., Phys. Plasmas, 17, 122701 (2010) 